\title{
Impact of diabetes and defective thyroid ontogenesis on audition
}

\section{Opinion}

Among all of the systemic diseases, metabolic pathologies have shown significantly damaging effects on the auditory system, due to the peculiarity of how their different mechanisms directly affect the morphogenesis and/or physiology of the auditory system. Among the diverse metabolic diseases, congenital hypothyroidism $(\mathrm{CH})$ and diabetes mellitus type I (DM1) are those most frequently related to the lesions that affect the peripheral and central auditory pathways, because of their physiopathological characteristics, and the high prevalence in the child population, in a critical period for overall human development. $\mathrm{CH}$ is one of the most common endocrine disorders in newborn infants (NI), affecting approximately 1:3000 to $1: 4000 \mathrm{NI}$ worldwide. ${ }^{1}$ The clinical manifestations are diverse and change according to the age group. In untreated $\mathrm{CH}$, somatic growth and functional differentiation of the central nervous system (SNC) are deep affected. ${ }^{2}$ The synthesis of various proteins and enzymes is subject to normal functioning of the thyroid gland, whose hormones are necessary for the formation of the middle and internal structures of the ${ }^{3}$ and of the central pathways of the auditory system. ${ }^{4}$ Therefore, in situations of hypothyroidism, especially the congenital form of the disease, it is possible for auditory damage of endocochlear, retrocochlear origin, and of the more central auditory portions to occur. $^{4}$

On the other hand, the prevalence of DM1 is more accentuated in early childhood; it is ranked the second most frequent chronic disease in this age group, and may affect adolescents and young adults. Its incidence is close to 0.5 new cases per every 100,000 inhabitants/ per year. ${ }^{5}$ After onset of the disease, important disturbances in the metabolism of carbohydrates, lipids and proteins develop, favoring the appearance of complications such as neuropathy and angiopathy, common in diabetes mellitus. ${ }^{6}$ In the auditory system, angiopathy may directly or indirectly interfere in the blood supply of the cochlea. But when neuropathy affects important sensory structures, it may cause auditory disturbances at the level of the peripheral or central pathways. ${ }^{7}$ Furthermore, damage may occur during treatment, such a conditions of chronic or intermittent hyperglycemia that are frequently related to the pathogenesis of the endothelial lesions in diabetes. ${ }^{8}$ Therefore, both metabolic alterations have the potential to injure the auditory structures, causing negative impacts on the affected person's life. Children with hearing impairment are unable to acquire oral language skills in the period or at an adequate speed, because the acquisition of oral language is an essentially auditory event. When hearing loss is detected early, even during the neonatal period, treatment may be performed, thus avoiding harmful damage to the child.

In view of the foregoing, auditory evaluation forms part of a
Volume 3 Issue 7 - 2016

\author{
Luciene da Cruz Fernandes, ${ }^{1,2}$ Caio Leonidas \\ Andrade ${ }^{3}$ \\ 'Department of Phoaudiology, University Federal of Bahia, Brazil \\ ${ }^{2}$ Department of Lifescience, Bahia State University, Brazil \\ ${ }^{3}$ Doctorate in Interactive Processes of Organs and Systems, \\ University Federal of Bahia, Brazil
}

Correspondence: Luciene C Fernandes, Department of Phonoaudiology, Av. Miguel Calmon, s/n, Salvador, Bahia, Brazil. Tel 557 I 32838 887, Email luenefernandes@gmail.com

Received:November II, 2016 | Published: November 25, 2016

battery of complementary exams when childhood metabolic diseases such as $\mathrm{CH}$ and DM are diagnosed. Thus, early intervention will favor the quality of life of these individuals.

\section{Acknowledgements}

None.

\section{Conflict of interest}

Author declares that there is no conflict of interest.

\section{References}

1. Setian N. Hypothyroidism in children: diagnosis and treatment. J Pediatr. 2007;83:209-216.

2. Rovet JF. Congenital hypothyroidism: an analysis of persisting deficits and associated factors. Child Neuropsychol. 2002;8(3):150-162.

3. Uziel A, Marot M, Rabie A. Corrective effects of thyroxine on cochlear abnormalities induced by congenital hypothyroidism in the rat. II. Electrophysiological study. Brain Res Rev. 1985;351(1):123-127.

4. Di Lorenzo L, Foggia L, Panza N, et al. Auditory brainstem response in thyroid diseases before and after therapy. Horm Res. 1995;43(5):200-205.

5. Diretrizes da Sociedade Brasileira de Diabetes (SBD). 2009.

6. Belan I, Passos JY. Clinical Nursing: pathophysiological and psychosocial approaches. Associated with Diabetes Mellitus. New York, USA; 1981;2:1037-1077.

7. Maia CAS, Campos CAH. Diabetes Mellitus como causa de perda auditiva. Braz J Othorrinolaryngol. 2005;71(2):208-214.

8. Thomas E. Hyperglycemia and insulin resistance: possible mechanism Academy of Sciences. New York, USA; 2002;8:967-943. 